\title{
DOAÇÃO DE ÓRGÃOS: A IMPORTÂNCIA DA COMUNICAÇÃO NA ESFERA FAMILIAR
}

\author{
ORGAN DONATION: THE IMPORTANCE OF COMMUNICATION IN \\ THE FAMILY BALL
}

\author{
Thayner Lacerda Lima ${ }^{1}$ \\ Macerlane de Lira Silva ${ }^{2}$ \\ Renata Lívia Silva Fonseca Moreira ${ }^{3}$ \\ Paulo Antônio Farias Lucena ${ }^{4}$
}

\begin{abstract}
RESUMO: INTRODUÇÃO: o transplante de órgãos pode ser a única alternativa terapêutica para pacientes portadores de doenças terminais, sendo a doação post mortem a forma mais frequente. No Brasil, a legislação vigente determina que a decisão sobre a doação de órgãos após a morte cabe aos familiares ou responsáveis legais do paciente. Apesar de ser o segundo maior transplantador do mundo, sua maior taxa de insucesso concentra-se na recusa dos familiares. Um dosmotivos frequente dessa negativa é o desconhecimento da opinião das pessoas, quando em vida, acerca da doação, demonstrando grande fragilidades no diálogo familiar dessa temática. OBJETIVO: evidenciar a relevância da comunicação no ambiente familiar, vislumbrando o consentimento positivo às custas da manifestação em vida dos pacientes sobre o desejo de ser doador. MÉTODOS: trata-se de um estudo de campo, transversal, descritivo, explicativo, observacional, com abordagem quantitativa de dados coletados através de questionário semiestruturado aplicado aos familiares de pacientes admitidos na Unidade de Terapia Intensiva e Sala Vermelha do Hospital Regional de Cajazeiras-PB. RESULTADOS: verificou-se que a maioria dos familiares desconhece a opinião dos pacientes sobre o processo de doação, corroborando como fato das pessoas não dialogarem sobre o tema na esfera familiar ou ainda serem contrários a esse processo. Em consonância com diversos estudos, houve o fortalecimentodeste como um dos principais conflitos que permeiam a negativa dos familiares, o que contribui para manter discrepante a
\end{abstract}

\footnotetext{
1 Discente de Bacharelado em Medicina da Faculdade Santa Maria - FSM.

2 Docente de Bacharelado em Medicina da Faculdade Santa Maria - FSM.

3 Docente de Bacharelado em Medicina da Faculdade Santa Maria - FSM. Enfermeira pela Universidade Federal da Paraíba - UFPB. Mestre em Enfermagem pela Universidade Federal da Paraíba - UFPB. Doutora em Pesquisa em Cirurgia pela Faculdade de Ciências Medicas Santa Casa de São Paulo - FCMSP.

4 Docente de Bacharelado em Medicina da Faculdade Santa Maria - FSM. Especialista em Neurologia. Mestre em Ciências da Saúde.
} 
demanda e a oferta de transplantes de órgãos. CONCLUSÃO: a partir dos resultados obtidos, ressalta-se a importância do diálogo sobre o assunto junto aos familiares, visto que conhecer a vontade do paciente facilita a tomada de decisão por seus responsáveis. Alternativas que favoreçam essa comunicação sobre o desejo da doação devem ser avaliadas e realizadas com maior enfoque, afim de esclarecer a população e diminuir as negativas devido à recusa familiar, vislumbrando a redução da enorme lista de espera por transplantes.

Descritores: Comunicação. Família. Obtenção de Tecidos e Órgãos. Transplante de Órgãos.

ABSTRACT: INTRODUCTION: Organ transplantation may be the only therapeutic alternative in terminally patients and postmortem donation is the most frequent form. In Brazil, current legislation determines that the decision on organ donation after death is up to the patient's family or legal guardians. Despite being the second largest transplant in the world, its highest failure rate is concentrated in family refusal. One of the frequent reasons for this refusal is the ignorance of people's opinion, when in life, about the donation, showing poor communication in the family dialogue of this theme. OBJECTIVE: To highlight the relevance of communication in the family environment, envisaging positive consent at the expense of patients' manifestation in life about the desire to be a donor. METHODS: This is a cross-sectional, descriptive, explanatory, observational field study with a quantitative approach to data collected through a semi-structured questionnaire applied to the relatives of patients admitted to the Intensive Care Unit and Red Room of the Cajazeiras-PB Regional Hospital. RESULTS: It was found that most family members are unaware of the patients' opinion about the donation process, corroborating with the fact that people do not talk about the theme in the family sphere or even oppose this process. In line with several studies, this was strengthened as one of the main conflicts that permeate the negative of family members, which contributes to keep the demand and supply of organ transplants discrepant. CONCLUSION: From the results obtained, the importance of dialogue on the subject with family members is emphasized, since knowing the patient's wishes facilitates decision-making by their caregivers. Alternatives that favor this communication about the desire for donation should be evaluated and made with greater focus in order to clarify the population and reduce the negative due to family refusal, to reducing the huge waiting list for transplants.

Descriptors: Communication. Family. Organ Transplantation. Tissue and Organ Procurement. 


\section{INTRODUÇÃO}

O transplante de órgãos pode ser a única alternativa terapêutica em pacientes portadores de doenças terminais de diferentes órgãos (WESTPHAL, 2016) e a doação post mortem é a forma mais frequente, sendo realizada após a confirmação do diagnóstico de morte encefálica $(\mathrm{ME})$, mediante consentimento familiar, de acordo com a Lei 9.434/1997. No contexto das políticas de saúde do Brasil, as relacionadas à doação de órgãos têm sido uma das mais trabalhadas, às custas de um grande impasse na estatística discrepante entre demanda e oferta de órgãos, proporcionando impacto positivo, porém, ainda necessitando da compreensão da população acerca dessa temática para que reduza essa discrepância (FELIX; SANTOS, 2015).

Historicamente, no Brasil, a Portaria 1.183/2000 discorria sobre a obrigatoriedade do registro da manifestação de desejo de ser doador ou não nas carteiras de identidade e de habilitação, logo em seguida substituído pelo Registro Nacional de Doadores. Em 2001, a Lei 10.211 modificou a Lei 9.434/1997 sobre a remoção de órgãos, tecidos e partes do corpo humano para fins de transplante e, a partir de então, o que constava nesses documentos, a doação presumida, passou a ser desconsiderada em detrimento da doação consentida pela vontade familiar. Portanto, tornou-seainda mais importante o paciente manifestar o seu desejo de doar para familiares e parentes. Essas modificações foram justificadas pela exigência das equipes transplantadoras sempre consultarem os familiares sobre a autorização da doação, independente de documentação comprobatória de manifestação positiva do potencial doador (ROCHA et al., 2016).

A legislação vigente determina que a decisão sobre a doação de órgãos após a morte somente deverá ser solicitada aos familiares ou responsáveis legais do paciente após concluído o diagnóstico da morte encefálica (ME), realizada a comunicação clara da situação a eles e afastadas as contraindicações para a doação (BRASIL, 2017). Nesse cenário, a família consiste em elemento principal, e 
apesar do Brasil ser o segundo maior transplantador do mundo, atrás apenas dos Estados Unidos, sua maior taxa de insucesso concentra-se na recusa dos familiares na obtenção de órgãos (FELIX; SANTOS, 2015; NAZÁRIO, 2019; GOIS, 2017).

A negativa familiar ocupa o primeiro lugar nas causas de não concretização depotenciais doadores, correspondendo a 41\% no primeiro semestre de 2019 . Dentre as motivações explicitadas pelas famílias para a recusa, é frequente o desconhecimento acerca do desejo do falecido ou, quando em vida, ser contrário à doação. Esses fatos ressaltam a importância do diálogo sobre a doação de órgãos na esfera familiar, visto que conhecer o desejo do paciente facilita a tomada de decisão por parte dos seus responsáveis (GOIS, 2017).

A fim de estimular cada vez mais as pessoas a serem doadoras, bem como reforçar a necessidade de informar à família o desejo de ser um doador, alguns órgãos criaram campanhas com enfoque nessacomunicação. A exemplo disso, o Ministério da Saúde comemora o Dia Nacional da Doação de Órgãos no dia 27 de setembro por meio da Campanha "Setembro Verde", instituída pela lei $n^{\circ}$ 15.463/14 que definiu esse mês a fim de ampliar o incentivo à doação de órgãos (ROCHA et al., 2016; ABTO, 2019).

Assim, o objetivo deste estudo consiste em reforçar a relevância da comunicação intrafamiliar, vislumbrando o consentimento familiar positivo às custas, principalmente, da informação por parte das pessoas, aqui representados por pacientes do Hospital Regional de Cajazeiras - PB (HRC), durante a vida, do desejo de ser doador. Dessa forma, almeja-se ampliar a discussão desse tema e reduzir uma das principais fragilidades que englobam esse processo e insistem em manter uma significativa lista de espera por transplantes de órgãos.

\section{MATERIAIS E MÉTODOS}

Trata-se de um estudo de campo, de caráter descritivo, explicativo e observacional, a partir de uma análise transversal, ou seja, realizada em um determinado momento, com abordagem quantitativa e sistematizada de dados 
coletados com a utilização de parâmetros estatísticos para a classificação e análise (FONTELLES et al., 2009).

A pesquisa de campo procura aprofundar as questões propostas a partir da observação de fatos tal como ocorrem e da análise de informações que buscam responder uma hipótese, verificar novos fenômenos ou relações entre eles, contribuindo para o desenvolvimento de uma consciência crítica, interpretadas através de uma reflexão de caráter científico,o que reforça sua natureza descritiva, sem interferência do pesquisador (MARCONI; LAKATOS, 2010; MANZATO; SANTOS, 2012).

Com o intuito de identificar fatores que expliquem a ocorrência dos fenômenos desse estudo, a pesquisa explicativa o complementa por meio de um conhecimento mais aprofundado da realidade em busca de uma relação entre causa e efeito (GIL, 2008; PRADANOV; FREITAS, 2013).

O cenário do estudo compreendeu as salas de espera dos setores da Unidade de Terapia Intensiva (UTI) e doEixo Vermelha do HRC, hospital que atende cerca de 167 mil habitantes com assistência de média complexidade. Utilizou-se de técnicas padronizadas para a coleta de dados, como a entrevista, aplicação de um questionário semiestruturado direcionado aos familiares dos pacientes admitidos nessas unidades, contendo perguntas norteadoras pertinentes ao objetivo proposto nesse estudo.

Por envolver seres humanos, a coleta dos dados foi realizada somente após aprovação do Comitê de Ética em Pesquisa da Faculdade Santa Maria, em consonância com as recomendações da Comissão Nacional de Ética em Pesquisa (CONEP), expressas na Resolução n 466 de 2012 do Conselho Nacional de Saúde (CNS) , do Termo de Anuência autorizado pelo Núcleo de Educação Permanente em Saúde do HRC, e das assinaturas dos Termos de Consentimento Livre e Esclarecido (TCLE) por cada indivíduo que aceitou participar da pesquisa após esclarecimento da temática, benefícios previstos, potenciais riscos e incômodo que esta pudesse acarretar. O Termo garantiu autonomia, anonimato e autorização da participação voluntária na pesquisa, além de suspensão imediata da pesquisa e oferta de apoio psicológico em caso de qualquer desconforto ou constrangimento. 
A população foi constituída por 48 familiares que estiveram acompanhando os pacientes admitidos e internados nos respectivos setores no período de abril a junho de 2018. A definição aproximada desse quantitativo foirealizada por uma amostra probabilística, a partir de informações do Banco de Dados do HRC, tomando-se como base uma média do número de admissões mensais nas referidas unidades.Foram considerados critérios de inclusão: acompanhantes responsáveis pelo paciente nesses setores, idade acima de 18 anos, boas condições psicológicas e disposição para participação voluntária na pesquisa;e excluídos do estudo aqueles que não aceitaram participar ou não estavam em boas condições psicológicas. As internações que seguiram o fluxo de complexidade da assistência, do Eixo Vermelho II para a UTI, compuseram a pesquisa apenas uma vez.

\section{RESULTADOS}

A partir da verificação dos questionários aplicados, foi possível realizar uma análise descritiva do perfil sociodemográfico da amostra (Tabela 01). Dos 48 participantes incluídos no estudo, $70,8 \%$ eram do sexo feminino e $29,1 \%$ do sexo masculino. Em relação a faixa etária, que variou dos 18 aos 70 anos, os menores de 25 anos corresponderam a 8,4\%, dos 25 aos 40 anos e maiores de 40 anos com taxas semelhantes de $48,5 \%$ cada um destes grupos. Quanto à moradia, a maioria residia na zona urbana contabilizando $68,7 \%$ e $31,3 \%$ na zona rural. 
Tabela 01: perfil sociodemográfico da amostra.

\begin{tabular}{llc}
\hline \multicolumn{1}{c}{ Variável } & \multicolumn{1}{c}{ Categoria } & $\%(\mathbf{N})$ \\
\hline Sexo & Feminino & $70,8 \%(34)$ \\
& Masculino & $29,1 \%(12)$ \\
Faixa etária & & \\
& Menores de 25 anos & $8,4 \%(4)$ \\
& $25-40$ anos & $45,8 \%(22)$ \\
Moradia & Maiores de 40 anos & $45,8 \%(22)$ \\
& & \\
& Zona urbana & $68,7 \%(33)$ \\
& Zona rural & $31,3 \%(15)$ \\
\hline
\end{tabular}

Fonte: elaborado pelos pesquisadores, 2019.

Em referência ao objetivo desse estudo, os familiares foram questionados se os respectivos pacientes já haviam expressado algo acerca da doação de órgãos (tabela 02). A maioria respondeu que esses nunca haviam comunicado algo a respeito, correspondendo a $87,5 \%$ das respostas. Apenas $12,5 \%$ tinham relatado sua vontade quanto à doação, desses, $66,6 \%$ positivos e $33,3 \%$ contrários ao ato.

Tabela 02: questionário aplicado aos familiares acerca da comunicação dos pacientes sobre doação.

\begin{tabular}{lcc}
\hline Questões & Respostas & $\%(\mathbf{N})$ \\
\hline 1) Expressaram sobre ser doador de órgãos & Sim & $12,5 \%(6)$ \\
& Não & $87,5 \%(42)$ \\
& & \\
2) Desejo de ser doador & Sim & $66,6 \%(4)$ \\
& Não & $33,3 \%(2)$ \\
\hline
\end{tabular}

Fonte: elaborado pelos pesquisadores, 2019.

\section{DISCUSSÃO}

Ao considerar que a legislação brasileira exige a autorização familiar para efetivar a retirada de órgãos e tecidos de um doador falecido,faz-se necessário 
discutir os fatores quese relacionam aos motivos da recusa familiar, visto que esta é a principal causa da não concretização de potenciais doadores. Em concordância com o que ocorre no Brasil, a Paraíba também conta com um número elevado na lista de espera, totalizando 468 indivíduos. Dos 65 potenciais doadores notificados nesse estado, no primeiro semestre de 2019, apenas 5 tornaram-se efetivos (ABTO, 2019).

Diversos estudos, ao abordarem sobre os conflitos que permeiam a negativa dos familiares, trazem como um dos motivos o fato das pessoas não dialogarem sobre doação no ambiente familiar (ALCÂNTARA et al., 2019). Culturalmente, a sociedade não é habituada a falar sobre a morte. Dessa forma, evita-se pensar na sua possibilidade e, consequentemente, não discorre acerca da sua opinião ou desejo, durante a vida, em relação a doação dos órgãos. Isso dificulta a tomada de decisão familiar quando necessário. Campanhas educativas e de divulgação sobre o tema se mostram uma ferramenta facilitadora (MARINHO et al., 2018).

Os resultados do presente estudo corroboram com o exposto, visto que a maioria dos familiares e responsáveis desconhecia o desejo quanto à doaçãodos respectivos pacientes em questão. Em relação ao quantitativo mínimo que havia compartilhado sua decisão, metade era contrário ao processo de doar seus órgãos. Essa negativa, previamente conhecida como causa da desaprovação familiar, também foi observada em trabalhos semelhantes, o que reforça a manutenção das filas de espera por transplante (BERTASI et al., 2019; OLIVEIRA, 2018; SOUSA, 2018).

Dessa forma, ainda no meio científico, artigos evidenciam o interesse positivo à doaçãodas pessoas, manifestado em vida, facilita o posicionamento dos familiares, pois estes tendem a ser favoráveis ao desejo dos seus entes falecidos. Tal comportamento pode contribuir para maior segurança dos familiares no momento decisivo da doação e reduzir os índices de recusa familiar (OLIVEIRA, 2018; MAYNARD et al., 2015).

Deparando-se com o mesmo cenário, pesquisadores brasileiros discutem alternativas que poderiam reduzir a enorme demanda por transplantes órgãos. Citaram, durante a pesquisa, a existência em outros países, como Alemanha e Holanda, de sistemas de cadastro de doadores, nos quaisas pessoas registram seu 
desejo de doação, auxiliando a família para uma decisão favorável quando necessário (BERTASI et al., 2019). No Brasil, por exemplo, o Programa Estadual de Transplante (PET) no Rio de Janeiro otimizou a comunicação com a populaçãoatravés de sites, Disque-Transplante (155), cartilhas, palestras e pela campanha "Doe+Vida". Essa campanha também conta com um siteno qual as pessoas podem se cadastrar e imprimir um cartão virtual do doador, afim de enfatizar a importância de expressar o desejo quanto à doação de órgãos e tecidos e a comunicação acerca do seu desejo (ROCHA et al., 2016).

\section{CONCLUSÃO}

Ao analisar os resultados provenientes dos questionários dos participantes entrevistados nesse estudo, os quais constituíam os familiares e responsáveis legais dos pacientes, observou-se que a maioria realmente não conhecia a opinião destes últimos acerca da doação de órgãos e tecidos. Portanto, no atual contexto sociolegal, é de grande relevância conhecer a vontade do doador, como importante fator de interferência na efetivação da doação de órgãos, visto que, em consonância com diversos estudos, a família tende a considerá-la.

Nesse sentido, identificar os motivos que envolvem a recusa pode contribuir para respaldar o planejamento de ações visando a promoção da doação de órgãos e tecidos, colaborando, deste modo, para a redução da discrepância existente entre a lista de espera e de oferta. Ressalta-se, então, a necessidade do debate contínuo perante a sociedade, enfatizando a importância da opinião e do conhecimento populacional sobre essa temática, às vistas da sua influência diretanesse processo de captação de órgãos.

Campanhas educativas e de divulgação sobre o tema compreendem ferramentas facilitadoras. Entre alternativas, o estabelecimento de um registro de doadoresnos sistemas de saúde poderia ser passível de avaliação pelas legislações vigentes. 
Sugere-se,por intermédio dessa pesquisa, proporcionar o desenvolvimento de outros estudos discorrendo sobre o assunto, bem como nortear políticas que se empreguem na dispersão de dados a respeito da doação de órgãos, propiciando discussões na população, principalmente em relação as causas de recusa familiar, a fim de reduzir seus altos índices. Assim, a população pode entender a necessidade dos vários indivíduos que esperam por um ato de solidariedade nas filas de transplantes.

\section{REFERÊNCIAS BIBLIOGRÁFICAS}

ABTO - Associação Brasileira de Transplantes de Órgãos. Registro Brasileiro de Transplantes. Dados Numéricos da doação de órgãos e transplantes realizados por estado e instituição no período: janeiro / junho - 2019. Ano XXV, nº 2. São Paulo, 2019.

ALCÂNTARA, Fabíola de Oliveira et al. Prevalência da recusa familiar quanto a doação de órgãos para transplante no estado de Rondônia. Revista Eletrônica Acervo Saúde, n. 34, p. e1014-e1014, 2019.

AMARAL, Adaíse Passos Souza et al. Desafios encontrados no processo de doação de órgãos: relato de experiência. Lecturas: Educación Física y Deportes, v. 23, n. 244, p. 86-97, 2018.

BERTASI, Raphael Adroaldo de Oliveira et al. Perfil dos potenciais doadores de órgãos e fatores relacionados à doação e a não doação de órgãos de uma Organização de Procura de Órgãos. Revista do Colégio Brasileiro de Cirurgiões, v. 46, n. 3, 2019.

BRASIL.Conselho Federal de Medicina. Resolução CFM n² 2173, de 23 de novembro de 2017. Brasília: CFM; $2017 . \quad$ Disponível em https://sistemas.cfm.org.br/normas/visualizar/resolucoes/BR/2017/2173.

BRASIL. Conselho Federal de Medicina. Resolução CFM no 1.480, de 8 de agosto de 1997 [Internet]. Dispõe sobre a caracterização de morte encefálica. Brasília: CFM; 1997. Disponível em: <http://www.portalmedico. org.br/resolucoes/CFM/1997/1480_1997.htm>. Acesso em: 29 set. 2017.

BRASIL.Ministério da Saúde. Conselho Nacional de Saúde. Resolução n $^{\circ}$ 466, de 12 de dezembro de 2012. Aprova as diretrizes e normas regulamentadoras de pesquisas envolvendo seres humanos. Diário Oficial da República Federativa do Brasil, 2013.

BRASIL. Ministério da Saúde. Lei 9.434, de 4 de fevereiro de 1997. Dispõe sobre a remoção de órgãos, tecidos e partes do corpo humano para fins de transplante e tratamento e dá outras providências. Brasília, $\quad 1997 . \quad$ DF, Disponível em: <http://dtr2001.saude.gov.br/sas/dsra/lei9434.htm>. Acesso em: 29 set. 2017.

FELIX, Micaella Bento; SANTOS, Walquiria Lene dos. Conhecimento da população do município de Valparaíso de Goiás sobre o processo de doação de órgãos para transplantes. Revista de Divulgação Científica Sena Aires, v. 4, n. 1, p. 3-10, 2015.

FONTELLES, Mauro José et al. Metodologia da pesquisa científica: diretrizes para a elaboração de um protocolo de pesquisa. Revista Paraense de Medicina, v. 23, n. 3, p. 1-8, 2009. 
GIL, Antonio Carlos. Métodos e técnicas de pesquisa social / Antonio Carlos Gil. - 6. ed. - São Paulo: Atlas, 2008.

GOIS, Renata Santos Silva et al. Efetividade do processo de doação de órgãos para transplantes. Acta Paulista de Enfermagem, v. 30, n. 6, p. 621-627, 2017.

MANZATO, Antonio José; SANTOS, Adriana Barbosa. A elaboração de questionários na pesquisa quantitativa. Departamento de Ciência de Computação e Estatística-IBILCEUNESP, p. 1-17, 2012.

MARCONI, Marina de Andrade; LAKATOS, Eva Maria. Fundamentos de metodologia científica. São Paulo: Atlas, 2010.

MARINHO, Christielle Lidianne Alencar; DE CARVALHO CONCEIÇÃO, Ana Isabel Cezário; DA SILVA, Rudval Souza. Causas de recusa familiar na doação de órgãos e tecidos. Revista Enfermagem Contemporânea, v. 7, n. 1, p. 34-39, 2018.

MAYNARD, Lorena Oliveira Dantas et al. Os conflitos do consentimento acerca da doação de órgãos post mortem no Brasil. Revista de Direito Sanitário, v. 16, n. 3, p. 122-144, 2015.

NAZÁRIO, Fernanda. Conscientização sobre doação de órgãos é primeiro passo para salvar vidas. Secretaria de Estado de Saúde de Mato Grosso. Desenvolvido pela Coordenadoria de Tecnologia de Informação. Cuiabá-MT, 20 de set. de 2019. Disponível em: <http://www.saude.mt.gov.br/noticia/5941>. Acesso em: 23 de out. de 2019.

OLIVEIRA, Kelen Caroline Lima de; NIHEI, Oscar Kenji. Doação de Órgãos: Fatores Dificultadores e Desafios. Revista Pleiade, v. 12, n. 23, p. 23-29, 2018.

PRODANOV, Cleber Cristiano; DE FREITAS, Ernani Cesar. Metodologia do Trabalho Científico: Métodos e Técnicas da Pesquisa e do Trabalho Acadêmico - $2^{\mathrm{a}}$ Edição. Editora Feevale, 2013.

RIBEIRO, Beatriz Camargo. ASPECTOS GERAIS DO TRANSPLANTE DE ÓRGÃOS: DOAÇÃO, PROIBIÇÃO DA COMERCIALIZAÇÃO E A IMPORTÂNCIA DO CONSENTIMENTO À LUZ DA DIGNIDADE DA PESSOA HUMANA. ETIC-ENCONTRO DE INICIAÇÃO CIENTÍFICA-ISSN 21-76-8498, v. 14, n. 14, 2018.

ROCHA, Rita de Cássia Machado da et al. Educação em Rede e possíveis contribuições para a Doação de Órgãos. Dissertação (Mestrado em Ensino em Biociências e Saúde) Fundação Oswaldo Cruz, Instituto Oswaldo Cruz, Rio de janeiro, RJ, 2016.

SOUZA, Edson Rosa de. Direito da pessoa de optar em vida pela doação (ou não) de seus órgãos post mortem. Direito-Tubarão, 2018.

WESTPHAL, Glauco Adrieno et al. Diretrizes para avaliação e validação do potencial doador de órgãos em morte encefálica. Rev. bras. ter. intensiva, v. 28, n. 3, p. 220-255, 2016. 